University of Nebraska - Lincoln

DigitalCommons@University of Nebraska - Lincoln

8-15-2007

\title{
Efficient angular dispersion compensation in holographic generation of intense ultrashort paraxial beam modes
}

James Strohaber

University of Nebraska-Lincoln, jstroha1@gmail.com

C. Petersen

University of Nebraska - Lincoln

Cornelis J. Uiterwaal

University of Nebraska - Lincoln, cuiterwaal2@unl.edu

Follow this and additional works at: https://digitalcommons.unl.edu/physicsuiterwaal

Part of the Physics Commons

Strohaber, James; Petersen, C.; and Uiterwaal, Cornelis J., "Efficient angular dispersion compensation in holographic generation of intense ultrashort paraxial beam modes" (2007). C.J.G.J. Uiterwaal Publications. 1.

https://digitalcommons.unl.edu/physicsuiterwaal/1

This Article is brought to you for free and open access by the Research Papers in Physics and Astronomy at DigitalCommons@University of Nebraska - Lincoln. It has been accepted for inclusion in C.J.G.J. Uiterwaal Publications by an authorized administrator of DigitalCommons@University of Nebraska - Lincoln. 


\title{
Efficient angular dispersion compensation in holographic generation of intense ultrashort paraxial beam modes
}

\author{
J. Strohaber,* C. Petersen, and C. J. G. J. Uiterwaal \\ Department of Physics and Astronomy, Behlen Laboratory, The University of Nebraska-Lincoln, City Campus, \\ Lincoln, Nebraska 68588-0111, USA \\ ${ }^{*}$ Corresponding author: jstroha1@bigred.unl.edu
}

Received May 17, 2007; accepted June 27, 2007;

posted July 16, 2007 (Doc. ID 83192); published August 3, 2007

\begin{abstract}
We experimentally demonstrate that small misalignments of the pulse stretcher or compressor of our chirped-pulse-amplification laser can precompensate for angular chirp when producing ultrashort paraxial beam modes with holographic gratings. Using this approach we can eliminate one of the two gratings needed in our $2 f-2 f$ setup [Mariyenko et al., Opt. Express 13, 7599 (2005)]. This allows for up to an order of magnitude more output power. We see our method as the next step in the production of intense exotic forms of ultrashort pulses, which can be used in the investigation of intense laser-matter interactions. In addition, we produce the first femtosecond (helical-)Ince-Gaussian beams. (C) 2007 Optical Society of America

OCIS codes: 050.1960, 090.1760, 050.1950, 140.3300, 320.7090.
\end{abstract}

Free-space paraxial modes are stationary-state (selfsimilar) solutions of the paraxial wave equation (PWE) for radiation in free space. The PWE is mathematically identical to the Schrödinger equation for the two-dimensional harmonic oscillator (2D-HO) [1-3]. The time-independent Schrödinger equation for the quantum 2D-HO is separable in three coordinate systems [4]: Cartesian, cylindrical, and elliptical. In the Cartesian case, the solutions are the Hermite-Gaussian (HG) modes [5]. In cylindrical coordinates the solutions are the Laguerre-Gaussian (LG) modes, which have received much attention because of their sharply defined amount of optical orbital angular momentum (OAM) [5-7]. The more recently found solutions of the PWE in elliptical coordinates $[8,9]$ are less well known; they are called Ince-Gaussian (IG) modes. Complex superpositions of the so-called even $\left(\mathrm{IG}^{e}\right)$ and odd $\left(\mathrm{IG}^{\circ}\right) \mathrm{IG}$ modes are referred to as (helical-)Ince-Gaussian (HIG) modes; like LG modes, they exhibit a nonzero amount of OAM.

We wish to create paraxial beams of ultrashort, intense pulses in a pure transversal mode to investigate the effect of OAM on light-matter interactions. Holographic diffraction techniques offer a flexible way to produce these transversal modes [7], but they cannot be applied directly to ultrashort pulses. Diffraction causes the different wavelengths in such pulses to travel in different directions, a phenomenon known as angular chirp. This leads to what is known as the chromatic effect [10]. Pulsed beams with angular chirp suffer from pulse front tilt and have a reduced bandwidth at any location in their transverse profile [11]. Because this lowers the focal intensity, angular chirp is undesirable for our purposes. Known schemes for holographic creation of beam modes that compensate for angular chirp are the "dispersionless $4 f$ " [12] and our " $2 f-2 f$ " [13] setups. A proof-ofprinciple experiment using a pulse compression scheme was carried out as an example of incorporating the existing compressor of a chirped-pulse- amplification (CPA) laser into the production of ultrashort LG beams [14]. Although this concept would allow for increased beam power, directly modifying the grating(s) in a CPA laser stretcher (or compressor) would be costly and inflexible. In this Letter we show that it is still possible to take advantage of the dispersive power of the stretcher (or compressor) grating(s) in a practical way and without altering these elements. Small misalignments of the stretcher (compressor) can lead to output beams possessing angular chirp [11]. We exploit this often unwanted angular chirp in the production of angular-chirp-free femtosecond paraxial beam modes. In the case of the stretcher, our method could be used in the exploration of optical mode amplification. Much research so far has focused on the production of angular-chirpfree, ultrashort LG beams. We show that our method also applies to other paraxial beam modes.

In the present work we use our $2 f-2 f$ scheme [13], but we eliminate its first grating, whose purpose was to precompensate for the angular chirp produced by the second grating. We show that this precompensation task can successfully be performed by a misaligned stretcher (or compressor). We find it convenient to think of a virtual grating that has replaced the original physical first grating. The grating constant and location of this virtual grating are then simply those that a real grating should have had to produce the same beam with a perfectly aligned stretcher (or compressor). The elimination of one real grating from our original $2 f-2 f$ setup makes it more energy efficient; e.g., by using binary gratings we observed an improvement of about an order of magnitude.

To verify that we indeed have a (virtual) grating source and to analyze its properties we consider a paraxial Gaussian beam with a $1 / e^{2}$ width $\omega(z)$ [5] diffracted by a line grating with grating constant $\Lambda$. We assume the spectrum of this beam is Gaussian as well, with central wavelength $\lambda_{0}$ and $1 / e^{2}$ width $\omega_{\lambda}$. The spectrum of the first-order diffraction beam is 
now a function of the transversal coordinate $x$ in the diffraction plane (i.e., there is angular chirp):

$$
\begin{aligned}
I(x, y, \lambda) \propto & \exp \left(-\frac{2 y^{2}}{\omega^{2}(z)}\right) \exp \left(-\frac{2 x^{2}}{\omega_{d}^{2}(z)}\right) \\
& \times \exp \left[-\frac{2 \omega_{d}^{2}(z)}{\omega^{2}(z) \omega_{\lambda}^{2}}\left(\lambda-\lambda_{0}-z C_{a} \frac{\omega_{\lambda}^{2}}{\omega_{d}^{2}(z)} x\right)^{2}\right] .
\end{aligned}
$$

Here $\omega_{d}(z)=\sqrt{\omega^{2}(z)+z^{2} C_{a}^{2} \omega_{\lambda}^{2}}$ is the $1 / e^{2}$ width of the intensity profile in the dispersion plane, and $C_{a}$ $=1 / \Lambda$ is the angular chirp. Equation (1) suggests two ways in which angular chirp can be measured. First, angular chirp causes the intensity profile to elongate in the $x$ direction upon propagation, and this can be measured using a CCD camera. Second, the spectrum of the diffracted beam has become a function of the position $x$ inside the beam. The central wavelength of the spectrum at position $x$ is $\lambda_{0}$ $+\left[z C_{a} \omega_{\lambda}^{2} / \omega_{d}^{2}(z)\right] x=\lambda_{0}+[m(z)] x$; we call $m(z)$ the spectral sweep. This latter quantity also contains angular chirp information and can be obtained using a spectrometer. Because the waist of our amplified beam was too large to be recorded with our CCD camera, we chose to determine the angular chirp of our virtual grating by measuring the spectral sweep $m(z)$.

In our first experiments (see Fig. 1) we slightly misaligned the stretcher (or compressor) in our Spectra-Physics Ti:sapphire Spitfire laser source and measured $m(z)$ at various distances $z$ from the last grating. The geometry of our stretcher is similar to the one described in [11], and we misalign it by displacing its retroreflector by a millimeter distance $\varepsilon$. Our compressor differs from the one described in [11] as it contains only one grating and two retroreflectors (instead of two gratings and one retroreflector). To misalign our compressor, we add a milliradian angle $\varepsilon_{t}$ to the $90^{\circ}$ opening angle of the first retroreflector. We measured misalignments using a digital micrometer with micrometer resolution. Wavelength spectra were recorded with a digital spectrometer whose input fiber we carefully positioned at various positions $x$ across the beam. Figure 1(a) shows the data measured for the stretcher with $\varepsilon \approx-21 \mathrm{~mm}$. Fitting this data to Eq. (1) we find for the angular chirp $C_{a} \approx 25 \mathrm{~mm}^{-1}$, or a virtual grating constant $\Lambda$
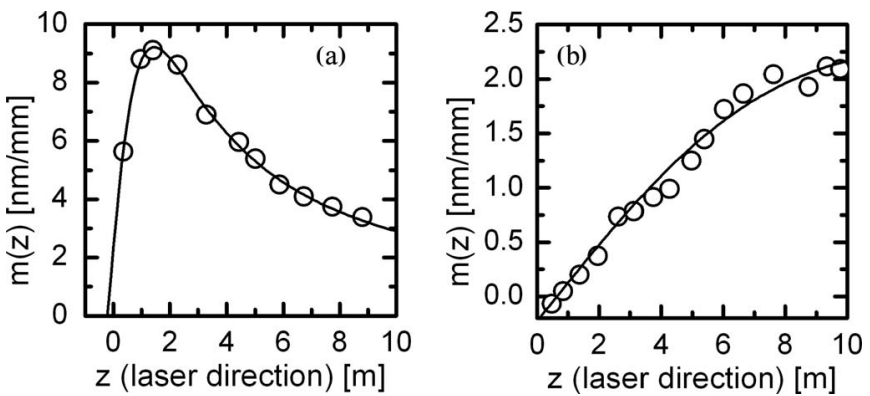

Fig. 1. Spectral sweeps $m(z)$ of the beam from misaligned (a) stretcher or (b) compressor; solid curves follow from Eq. (1) (see text). $\approx 40 \mu \mathrm{m}$. Figure $1(\mathrm{~b})$ was obtained with the misaligned compressor; for $\varepsilon_{t} \approx-3.3 \mathrm{mrad}$ we find $C_{a}$ $\approx 16 \mathrm{~mm}^{-1}$, or $\Lambda \approx 60 \mu \mathrm{m}$.

To demonstrate that we can properly control $C_{a}$ we also measured it as a function of $\varepsilon$ (or $\varepsilon_{t}$ ) at a fixed position $z$ from the stretcher (compressor). For both devices we found that $C_{a}$ depends linearly on $\varepsilon\left(\varepsilon_{t}\right)$. The stretcher data agree with [11] [solid curve in Fig. 2(a)]. The solid curve in Fig. 2(b) is our calculation of $C_{a}$ for our compressor. Note that $C_{a}$ can be positive or negative depending on which edge of the beam profile has the smallest wavelength.

To investigate the performance of a virtual grating in our $2 f-2 f$ configuration [13] we carefully aligned a $75 \mathrm{~cm}$ focal length lens and, as a second grating, our Hamamatsu PPM X8267 spatial light modulator (SLM; for $750-850 \mathrm{~nm}$ ) with the slightly misaligned stretcher (or compressor). The virtual grating was typically located at $\sim 10 \mathrm{~cm}$ preceding ( $\sim 60 \mathrm{~cm}$ after) the last physical grating (see Fig. 1). (An analysis of the location of the virtual grating will be published elsewhere. If the SLM could be positioned at this virtual grating's location we could eliminate the lens.) We programmed our SLM to produce an $\mathrm{HG}_{1,0}$ mode, using a phase-only, off-axis hologram with phaseamplitude encoding $[8,15]$. We chose this mode because (in the ideal case) any intensity appearing between its two lobes is a measure of the amount of angular chirp not compensated by the whole setup. For a virtual grating constant $\Lambda=1 / C_{a}$ and an SLM grating constant $\Lambda_{\text {SLM }}$ the far-field intensity in the diffraction order \pm 1 is of the form
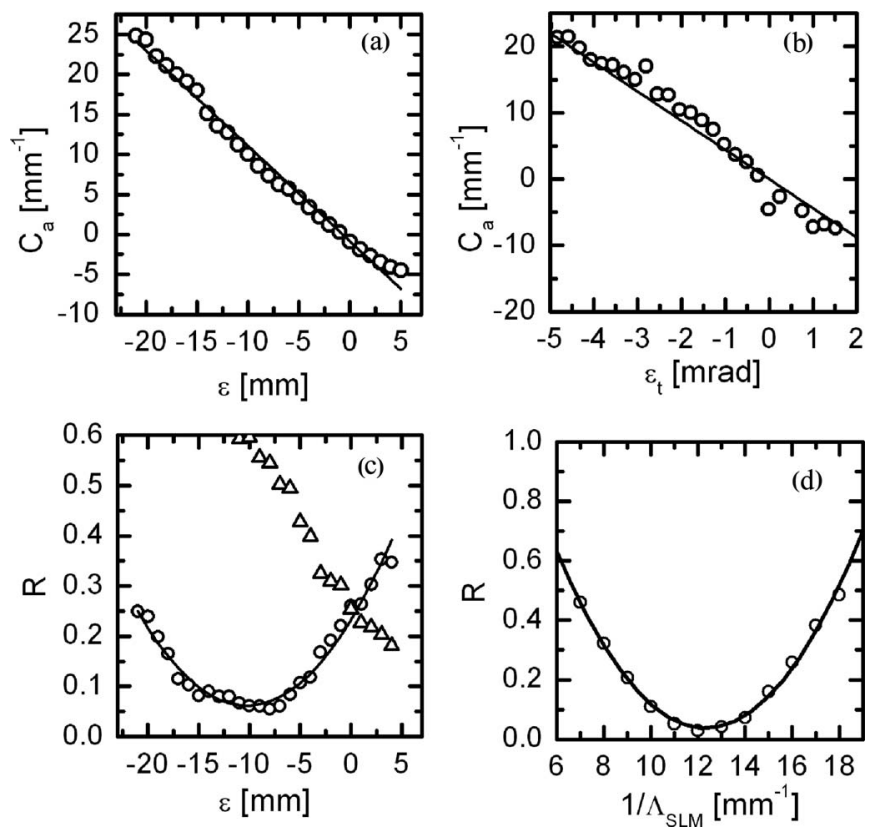

Fig. 2. (a) Angular chirp from misaligned stretcher versus $\varepsilon$. Circles, experiment; solid curve, theory. (b) Same as (a) for compressor, varying $\varepsilon_{t}$. (c) Intensity ratio $R$ measured with $\mathrm{HG}_{1,0}$ mode in stretcher- $2 f-2 f$ setup in the \pm 1 orders (circles-triangles) versus $\varepsilon$ for fixed $\Lambda_{\mathrm{SLM}}$. (d) $R$ for the compressor- $2 f-2 f$ setup in the +1 order versus $\Lambda_{\text {SLM }}$ for fixed $\varepsilon_{t}$. 


$$
\begin{aligned}
I(x, y)= & \int \mathrm{d} \lambda\left|\mathrm{HG}_{1,0}\left(x-\left(C_{a} \pm \frac{1}{\Lambda_{\mathrm{SLM}}}\right)\left(\lambda-\lambda_{0}\right) f, y\right)\right|^{2} \\
& \times \exp \left[-\left(\lambda-\lambda_{0}\right)^{2} / \omega_{\lambda}^{2}\right],
\end{aligned}
$$

where $f$ is the focal length of the lens used to image the far field. Upon performing the integral we find that the ratio $R$ of the residual intensity $I(0,0)$ between the modal lobes and the peak intensity of the lobes is quadratic in the residual chirp: $R$ $\propto\left(C_{a} \pm 1 / \Lambda_{\mathrm{SLM}}\right)^{2}$ (if this residual chirp is not too large). We used a $100 \mathrm{~cm}$ lens after the $2 f-2 f$ setup to measure $R$ with a CCD camera. For the stretcher we measured $R$ as a function of $\varepsilon$ [Fig. 2(c), circlestriangles are for the \pm 1 diffraction order]. For the compressor we recorded $R$ as a function of $\Lambda_{\mathrm{SLM}}$, keeping $\Lambda$ fixed [Fig. 2(d)]. In both cases the quadratic dependence was confirmed [solid curves in Figs. 2(c) and 2(d)]. The best compensations we achieved are $1-R=94 \%$ (stretcher) and 97\% (compressor). A percentage closer to the ideal value of $100 \%$ may be reached by improving the modal quality of the laser beam and by further optimizing the holographic grating on the SLM. For the compressor, varying $\Lambda$ while keeping $\Lambda_{\text {SLM }}$ constant was impractical because it requires repeated realignment. Instead, we recorded the \pm 1 diffraction order profiles (for the $\mathrm{HG}_{1,1}$ mode) for $C_{a}=+\Lambda_{\mathrm{SLM}}^{-1}$ (top row in Fig. 3), $C_{a}=0$ (middle row), and $C_{a}=-\Lambda_{\mathrm{SLM}}^{-1}$ (bottom row). The large degree of chirp compensation causes the crispness of Figs. 3(b) and 3(e). We repeated the compressor experiment with $C_{a}=+\Lambda_{\mathrm{SLM}}^{-1}$ for an assortment of HG, LG, and IG/HIG modes of femtosecond paraxial beams (Fig. 4). Frequency-resolved optical gating (FROG) measurements of a holographically generated $\mathrm{HG}_{0,0}$ beam in the properly compensated first diffraction order indicate pulse durations equal to that of our unmodified Spitfire output beam ( $\sim 43 \mathrm{fs})$. We conclude that the combination of the misaligned compressor and the SLM restores that original temporal phase. To the best of our knowledge, we produce the first femtosecond IG and HIG beams.

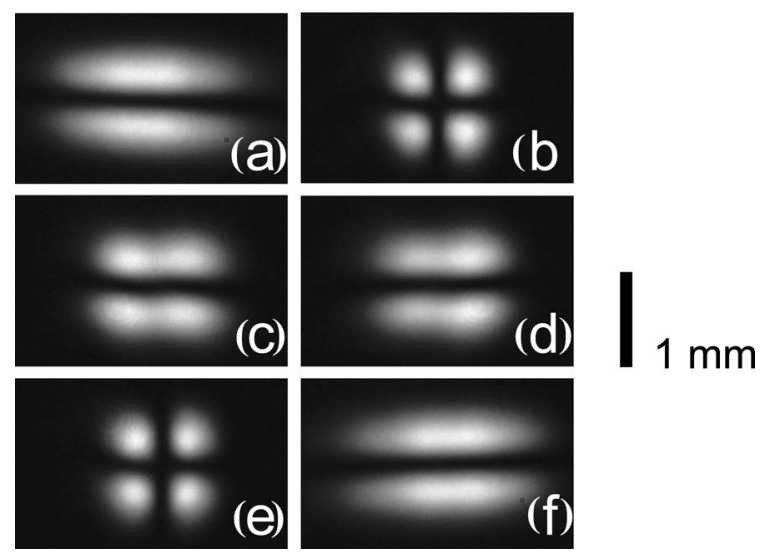

Fig. 3. Compressor- $2 f-2 f$ setup: Far-field profiles recorded in the \pm 1 diffraction order $\left(\mathrm{HG}_{1,1}\right.$ mode) for $C_{a}$ $=+\Lambda_{\mathrm{SLM}}^{-1}$ (top row), $C_{a}=0$ (middle row), and $C_{a}=-\Lambda_{\mathrm{SLM}}^{-1}$ (bottom row).
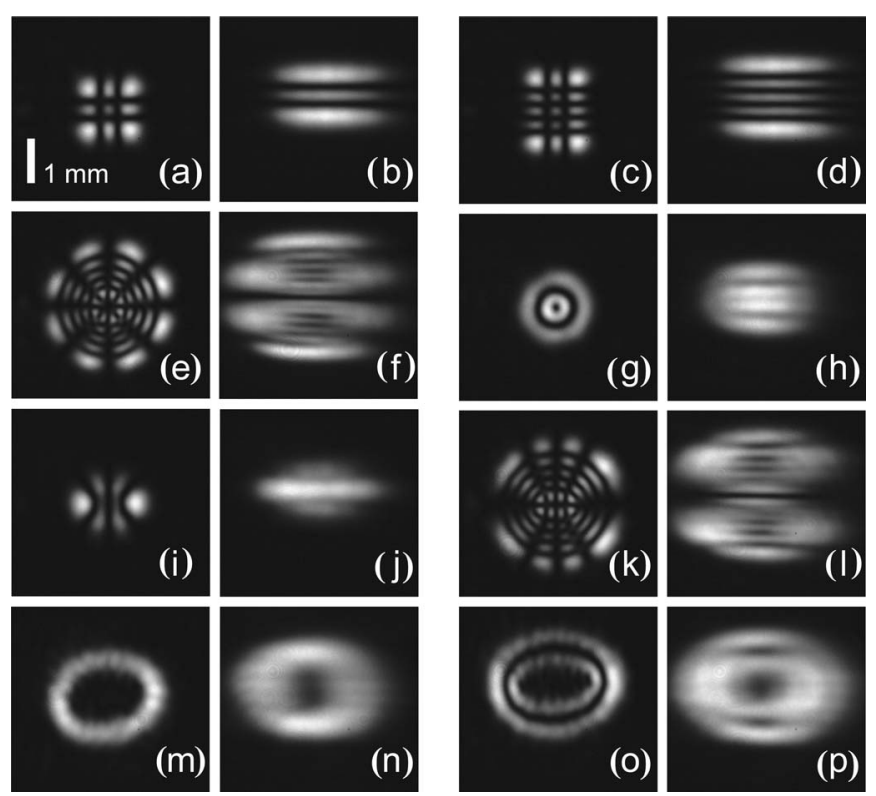

Fig. 4. Gallery of ultrashort paraxial modes created with our compressor- $2 f-2 f$ setup. Columns 1,3 , compensated order; columns 2, 4, uncompensated order. (a), (b) $\mathrm{HG}_{2,2}$, (c), (d) $\mathrm{HG}_{2,4}$, (e), (f) $\mathrm{LG}_{4,4}^{o}$, (g), (h) $\mathrm{LG}_{1,1}$ (i), (j) $\mathrm{IG}_{3,3,2}^{e}$, (k), (l) $\mathrm{IG}_{12,4,2}^{o},(\mathrm{~m}),(\mathrm{n}) \mathrm{HIG}_{10,10,4}^{+}$, (o), (p) $\mathrm{HIG}_{12,10,6}^{+}$.

In conclusion, we have demonstrated that small misalignments to a stretcher (compressor) can efficiently compensate for angular dispersion when producing ultrashort pulses in paraxial beam modes.

This material is based upon work supported by the National Science Foundation under grant PHY0355235 .

\section{References}

1. G. Nienhuis and L. Allen, Phys. Rev. A 48, 656 (1993).

2. O. Steuernagel, Am. J. Phys. 73, 625 (2005).

3. G.-Y. Tsaur and J. Wang, Phys. Rev. A 73, 033804 (2006).

4. C. P. Boyer, E. G. Kalnins, and W. Miller, Jr., J. Math. Phys. 16, 499 (1975).

5. A. E. Siegman, Lasers (University Science Books, 1986).

6. G. Molina-Terriza, J. P. Torres, and L. Torner, Nat. Phys. 3, 305 (2007).

7. L. Allen, S. M. Barnett, and M. J. Padgett, eds., Optical Angular Momentum (IOP, 2003).

8. M. A. Bandres and J. C. Gutiérrez-Vega, J. Opt. Soc. Am. A 21, 873 (2004).

9. J. B. Bentley, J. A. Davis, M. A. Bandres, and J. C. Gutiérrez-Vega, Opt. Lett. 31, 649 (2006).

10. J. Leach and M. J. Padgett, New J. Phys. 5, 154 (2003).

11. G. Pretzler, A. Kasper, and K. J. Witte, Appl. Phys. B 70, 1 (2000).

12. K. Bezuhanov, A. Dreischuh, G. G. Paulus, M. G. Schätzel, and H. Walther, Opt. Lett. 29, 1942 (2004).

13. I. G. Mariyenko, J. Strohaber, and C. J. G. J. Uiterwaal, Opt. Express 13, 7599 (2005).

14. K. Bezuhanov, A. Dreischuh, G. G. Paulus, M. G. Schätzel, H. Walther, D. Neshev, W. Królikowski, and Y. Kivshar, J. Opt. Soc. Am. B 23, 26 (2006).

15. J. A. Davis, D. M. Cottrell, J. Campos, M. J. Yzuel, and I. Moreno, Appl. Opt. 38, 5004 (1999). 